\title{
Performance Analysis of ADS-B Overlapping Signal Separation Algorithm based on RLS
}

\author{
Zhaoyue Zhang*
}

College of Air Traffic Management, Civil Aviation University of China, Tianjin, 300300, China

\begin{abstract}
In order to solve the problem of incorrectly decode the ADS-B signal and the missing aircraft information caused by overlap and interference, in the information transmission process of 1090ES ADS-B signal, a multi overlapping 1090ES ADS-B signal separation algorithm based on RLS algorithm is proposed. After a comprehensive analysis of the ADS-B signal to a plurality of base stations, the algorithm applies RLS blind source separation and recovers the source signals of ADS-B, thereby improving signal decoding accuracy and the dynamic performance monitoring for aircraft. The paper has verified the signal separation, including signals based on two level overlapped and three level overlapped and signals with noise, and verified the feasibility of RLS algorithm in ADS-B signal separation by MATLAB simulation test.
\end{abstract}

Key words: RLS; ADS-B; signal separation

(Submitted on December 9, 2017; Revised on January 16, 2018; Accepted on February 17, 2018)

(C) 2018 Totem Publisher, Inc. All rights reserved.

\section{Introduction}

1090ES ADS-B (Automatic Dependent Surveillance Broadcast) data link is the technology of aircraft integrated communication and monitoring, which can transmit aircraft status information, speed and altitude information [1]. However, ADS-B information is subject to various signal interference or signal overlap during the transmission process, and the ADS$B$ receiver over Satellite will receive more overlapped signal $[4,5]$. The overlapped signal has a great influence on the single ADS-B decoding device, resulting in the failure to correctly decode the ADS-B signal, resulting in the discontinuity of air traffic monitoring [2]. To solve this problem, the existing ADS-B receiving device is used as the original signal, and the correlation filtering algorithm is used to separate the signal, so as to improve the accuracy of decoding [3,7,9]. In this paper, the ADS-B overlapping signal separation algorithm based on RLS is proposed, and the experimental simulation and verification are carried out.

\section{ADS-B signal model}

The 1090ES ADS-B message includes 4 preamble pulse and 112 bits message sequences, and the message block format is coded with pulse position modulation (PPM), as shown in Figure 1 [6]. ADS - B message contains 4 recognition pulses, and each pulse duration is $0.5 \pm 0.05 \mu \mathrm{s}$. Each bit 1090ES ADS-B message sequence is accounted for $1 \mu$ s .In the $1 \mu$ s signal generation, decreasing signal is represented by binary "1", and rising signal is represented by binary " 0 ". The 4 preamble pulse of the front end is the reference voltage of the recognition level in the subsequent message, which is the basis of the decoding of the ADS-B signal.

\footnotetext{
* Corresponding author.

E-mail address: zy_zhang@cauc.edu.cn
} 


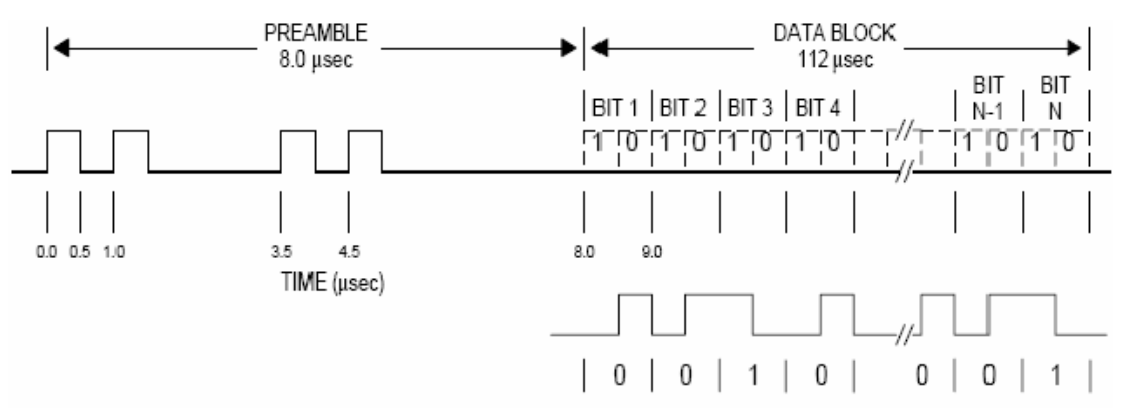

Figure 1. Transmission Waveform of ADS-B Message

In the field of communication, the crosstalk between signals is always a factor that affects the quality of communication. When multiple 1090ES ADS-B signals are propagating at the same time, there may be a case of partial pulse code overlapping. The current $\mathrm{S}$ mode error correction algorithm is mainly realized by locating the time window with low confidence, also known as "sliding time window". The clutter generated by the overlapping of multiple 1090ES ADS-B signals makes the subsequent 1090ES ADS-B decode a great deal of error. As shown in Figure 2, when the pulse signal 1 (gray) and the pulse signal 2 (white) appear simultaneously, the problem of overlapped signals will appear. After overlapping, the signals have a great influence on the leading pulse. In the subsequent decoding process, the reference of signal power is missing, resulting the error of signal decoding, so then the signal would discard the current 1090ES ADS-B information and lose the monitoring of the state of the aircraft. Aiming at the problem of ADS-B decoding, we use RLS algorithm to solve the signal overlapping, separate the overlapped signals of 1090ES ADS-B, finally separate and restore the initial pulse signal.

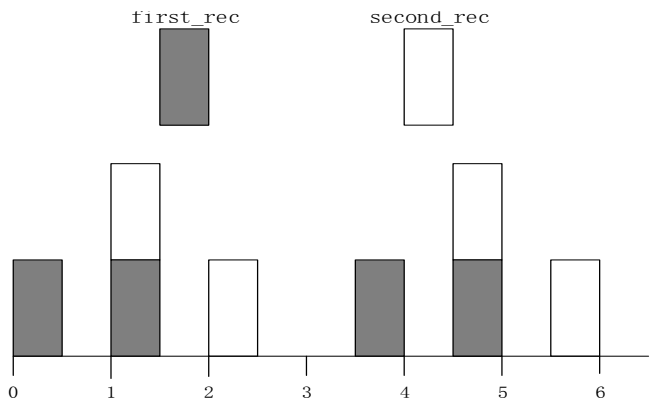

Figure 2. ADS-B Signal Superposition Model

According to the above 1090ES ADS-B signal model, every ADS-B base station receives the overlapped signals and has the characteristics of random combination. MATLAB is used as the simulation software to simulate the reception signal of ADS-B base station. Among them, the observation signal model of each ADS-B base station is a random superposition of multiple ADS-B source signals.

In the RLS algorithm, multichannel data received from multiple base stations of 1090ES ADS-B are used as observation signals. The ADS-B observation signals need to be stacked randomly by the ADS-B source. $s(t)$ represents the source signal, $s_{1}(t), s_{2}(t), s_{3}(t) \cdots s_{n}(t)$ is a signal sequence of an arbitrary $\mathrm{n}$ unit time. $x(t)$ represents the observation signal, $x_{1}(t), x_{2}(t), x_{3}(t) \cdots x_{m}(t)$ is used to indicate the mixed signal sequence of ADS-B base station received.

The $x(t)$ is randomly stacked with a random matrix $s(t)$, and the mathematical model of the pulse position modulation encoding of the message data is described as a Eq. (1):

$$
\left\{\begin{array}{c}
x_{1}(t)=a_{11} s_{1}(t)+\cdots+a_{1 n} s_{n}(t) \\
\vdots \\
x_{m}(t)=a_{m 1} s_{1}(t)+\cdots+a_{m n} s_{n}(t)
\end{array}\right.
$$

where, $a_{i j}$ is the unknown mixing coefficient in the ADS-B information transmission process. In the mixture of ADS-B signals, it is assumed that $a_{i j}$ is an unknown constant matrix. The signal separation of ADS-B is how to separate the source signal $s_{1}(t), s_{2}(t), s_{3}(t) \cdots s_{n}(t)$ from the mixed observation signal received by the ADS-B. There is a noise signal in the actual 
ADS-B observation signal. If the interference term is taken into account, the observed signal of ADS-B received by each base station can be expressed as a Eq. (2):

$$
X(t)=A S(t)+n(t)
$$

$X(t)=\left\{x_{1}(t), x_{2}(t) \cdots x_{m}(t)\right\}$ is m-dimensional ADS-B base station received signal, also known as the observation signal, is the algorithm model as the input information; $S(t)=\left\{s_{1}(t), s_{2}(t) \cdots s_{n}(t)\right\}$ is a vector concluded by n-dimensional independent ADS-B source signal, in the mixed ADS-B model, $S(t)$ is unknown variables or independent component. $n(t)$ is noise vector, and $A$ is a mixed matrix which is composed with the multiple unknown mixed coefficient $a_{i j}$.

The algorithm model of ADS-B signal separation needs to calculate the separation matrix $W$, and extract and recover the source signal $S(t)$ of ADS-B from the observed signals $X(t)$. Assuming that $Y(t)$ is the estimated signal of the ADS-B source signal, the separation system is expressed as a Eq. (3):

$$
Y(t)=W X(t)
$$

\section{RLS Algorithm}

RLS algorithm is recursive least square method. RLS algorithm is a method based on nonlinear principal component analysis. Based on the minimum mean square error algorithm, this algorithm estimates the sum of the revalued square error of the input signal. RLS algorithm has a fast convergence speed and is widely used in the analysis of time series.

Here, the ADS-B observation signal needs to be pre-whitened, such as Eq. (4) and Eq. (5), to reduce the redundancy between the features of each input signal, so that the components are linearly irrelevant [8].

$$
\begin{gathered}
\tilde{x}(t)=x(t)-E\{x(t)\} \\
v(t)=E\left\{\tilde{x}(t) \tilde{x}^{T}(t)\right\}^{-1 / 2} x(t)
\end{gathered}
$$

Then, the optimization is performed according to the following cost function to obtain Eq. (6):

$$
J(W(t))=\sum_{i=1}^{t} \beta^{t-i}\left\|v(i)-W(i) g\left(W^{T}(i-1) v(i)\right)\right\|^{2}
$$

where, $\beta \in(0,1)$ is the forgetting factor, $g$ is the non-linear transformation function.

In the mathematical model of ADS-B signal separation, the first thing is to initialize $W(0), P(0)$;

Adaptive filtering is performed for ADS-B observation signal, as shown in Eq. (7):

$$
y(t)=W(t-1) v(t)
$$

The gain vector of the model needs to be updated:

$$
\begin{gathered}
z(t)=g(y(t)) \\
h(t)=P(t-1) z(t) \\
m(t)=\frac{h(t)}{\left(\beta+z^{T}(t) h(t)\right)}
\end{gathered}
$$

Update inverse matrix: 
Update weight vector:

$$
P(t)=\frac{1}{\beta} \operatorname{Tri}\left[P(t-1)-m(t) h^{T}(t)\right]
$$

$$
W(t)=W(t-1)+m(t)\left[v^{T}(t)-z^{T}(t) W(t-1)\right]
$$

Where, $\operatorname{Tr} i[\cdot]$ is the forced symmetric process, the upper triangular part of the matrix transposed to the lower triangular part. $W(0)$ and $P(0)$ are unit matrices. $g$ needs to be selected according to different signals. (13):

To assess the difference between the ADS-B source and separation signals, the signal reduction error $E$ is defined as Eq.

$$
E=\sum_{i=1}^{n}\left(\sum_{j=1}^{n} \frac{\left|c_{i j}\right|}{\max _{k}\left|c_{i k}\right|}-1\right)+\sum_{j=1}^{n}\left(\sum_{i=1}^{n} \frac{\left|c_{i j}\right|}{\max _{k}\left|c_{k j}\right|}-1\right)
$$

Where, $c_{i j}$ is the element of the jth column of the ith row of the matrix $C=W A$. The higher the similarity between the signal after the separation and reduction and the original signal, the smaller the value of $E$. This signal reduction error is used to judge the convergence and stability of the RLS algorithm.

\section{Algorithm Simulation and Experiment}

In order to verify the feasibility of this algorithm in the separation of ADS-B signals, double signals and triple signals are selected for verification respectively. In the ADS-B signal separation algorithm, we select $g(y)=y-\tanh (y)$, forgetting factor $\beta=0.99$. In the simulation experiment, the ADS-B signal is sampled at $10 \mathrm{Mhz}$, and the sampled data is 500 samples. The signal received by each ADS-B base station is a random superposition of ADS-B signals, respectively.

\subsection{Double Signal Superposition Separation Verification}

In the double signal separation simulation, Figure 3 is ADS-B source signal. Using pulse code modulation, each bit signal is 10 samples. Two ADS-B signal header recognition signals are consistent and the message bits are randomly generated. The ADS-B source signals are superimposed by the random matrix $A$ as observation signals, as shown in Figure 4 . The observation signal has the problem that the power of the header is inconsistent, which is basically consistent with the overlapped signal in the actual sampling, and the decoded information is error information. The separated signals separated and calculated by the RLS algorithm are shown in Figure 5. Figure 6 shows the error analysis of the source signal and the separated signal. As the number of superposition increases, the error decreases gradually, which shows that the RLS algorithm has a fast convergence speed, can meet the requirement of fast signal separation of ADS-B, and has good steady-state performance.
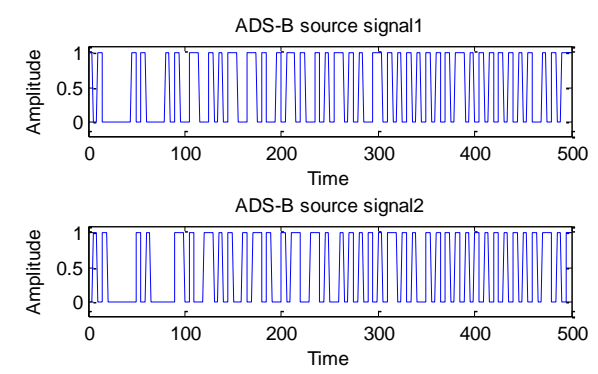

Figure 3. ADS-B source signals 

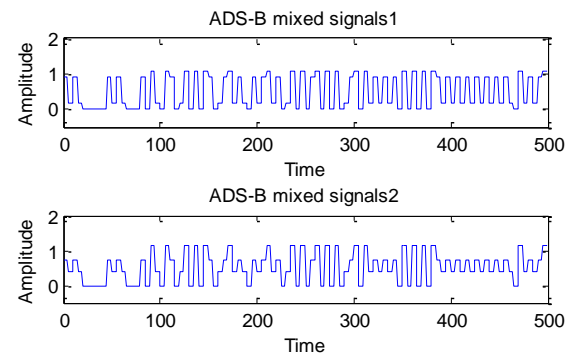

Figure 4. ADS-B mixed signals
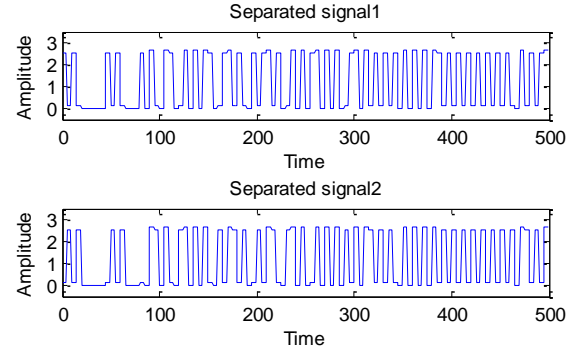

Figure 5. ADS-B separated signals

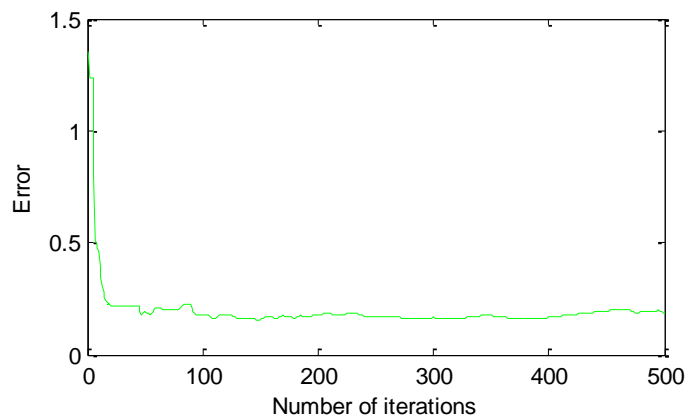

Figure 6. The error analysis of the source signal and the separated signal

\subsection{Triple Signal Separation Verification}

In the triple signal separation verification experiment, Figure 7 shows three ADS-B source signals generated randomly, and Figure 8 shows the observation signals generated by random superposition. As can be seen in Figure 8, the triple superposed signal is more complex than the double superposed signal, and the header and information part cannot be correctly identified, resulting in loss of ADS-B information. Figure 9 shows the source signals of ADS-B separated by RLS algorithm. The separated signals are basically the same as the source signals, and the leading pulse signal can also be used to identify and decode the reference. Figure 10 shows the error comparison between the source signal and the separated signal. As the number of iterations increases, the error decreases gradually. However, compared with double signal superposition, the convergence stability requires more iterations.
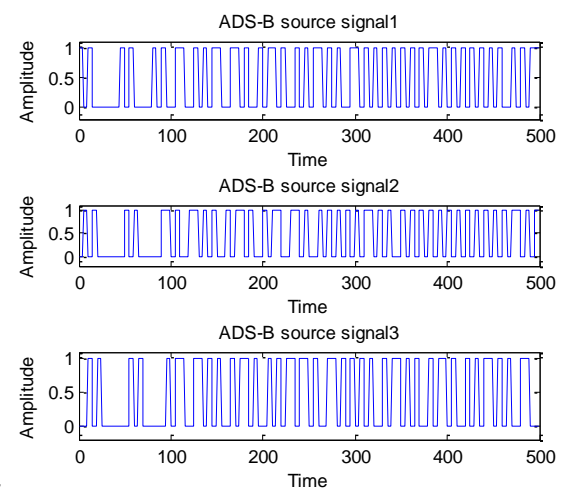

Figure 7. ADS-B source signals 

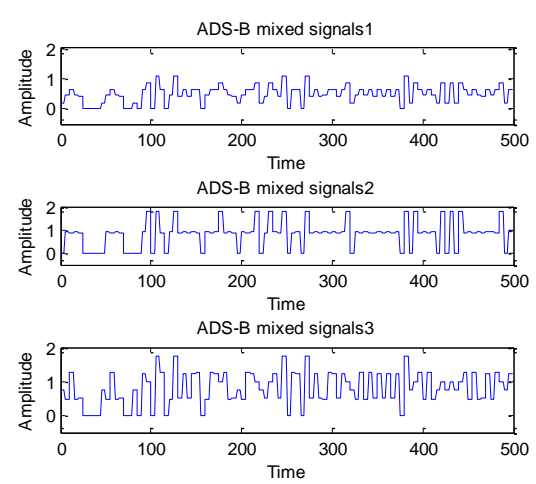

Figure 8. ADS-B mixed signals Separated signal1

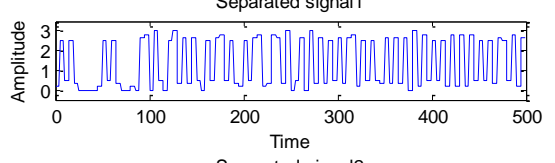

Separated signal2

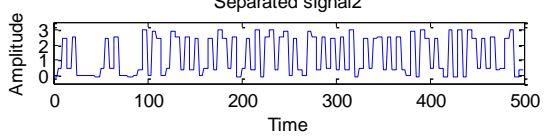

Separated signal3

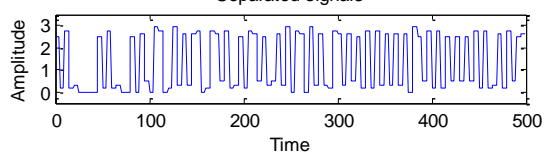

Figure 9. The separated signals

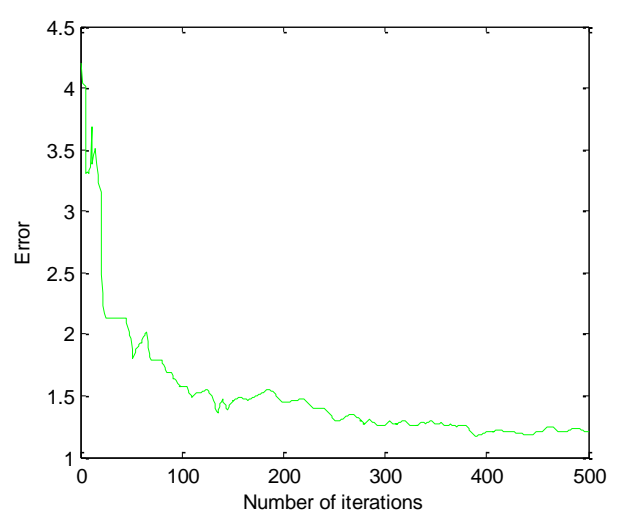

Figure 10. The error analysis of the source signal and the separated signal

\subsection{Simulation Results Analysis}

From the analysis of the simulation results, it can be concluded that the RLS algorithm can satisfy the separation of the ADSB multi-channel superposed signals and that the separated ADS-B signals are basically similar to the source signals, which can meet the requirements of ADS-B signal decoding. The error of the source signal and the separated signal of the double signal superposition model is 0.8203 ; the error of the source signal and the separated signal of the triple signal superposition model is 1.2075. After repeated experiments, the RLS algorithm can correctly separate the ADS-B mixed signals after adding noise interference, but there is randomness in the order of the output signals. After analyzing the error of the source signal and the separated signal, we can conclude that the RLS method has better convergence speed and higher steady-state characteristics, which is suitable for the processing of ADS-B signal with higher speed.

\section{Conclusions}

RLS algorithm is an efficient blind source separation algorithm. In this paper, aiming at the problem of multiple overlapping of ADS-B signals, the signals of multiple ADS-B signal base stations are used as observation signals, and the simulation and test are carried out by MATLAB, which verifies the feasibility of RLS algorithm in ADS-B signal separation. The 
experimental results show that the RLS algorithm can realize the recovery and reconstruction of ADS-B source signals and meet the decoding requirements of ADS-B.

\section{Acknowledgements}

This work was supported by the Special Funding Project of Civil Aviation University of China and the Central University (3122013Z006).

\section{References}

1. B. S. Ali, "System specifications for developing an Automatic Dependent Surveillance-Broadcast (ADS-B) monitoring system," International Journal of Critical Infrastructure Protection, vol. 15, pp. 40-46, December 2016.

2. B. S. Ali, W. Y. Ochieng, and R. Zainudin, "An analysis and model for Automatic Dependent Surveillance Broadcast (ADS-B) continuity," Gps Solutions, vol. 1, pp. 1-14, 2017.

3. S. Cruces-Alvarez, A. Cichocki, L. Castedo-Ribas, "An iterative inversion approach to blind source separation," IEEE Press, 2000.

4. T. Delovski, K. Werner, T. Rawlik, “ADS-B over Satellite The world's first ADS-B receiver in Space," in Proceedings of the Small Satellites Systems and Services Symposium, 2014.

5. R. V. D. Pryt and R. Vincent, "A Simulation of Signal Collisions over the North Atlantic for a Spaceborne ADS-B Receiver Using Aloha Protocol," Positioning, vol. 6, no. 3, pp. 23-31, 2015.

6. RTCA, "DO-260B: Minimum Operational Performance Standards for 1090 MHZ Extended Squitter Automatic Dependent Surveillance-Broadcast (ADS-B) and Traffic Information Services Broadcast (TIS-B),” 2013.

7. K. Y. Yan. K. Y, L. Ze-Jun, H. W. Shi, “An Improved Fast ICA ADS-B 1090ES Signal Separation Technique,” Computer \& Modernization, 2014

8. H. Yu, Z. Liu, "The Research on the Adaptive Algorithms of Blind Signal Separation," Computing Technology and Automation, vol. 4, pp. 76-79, 2008.

9. W. T. Zhang, S. T. Lou, Y. L. Zhang, "Robust nonlinear power iteration algorithm for adaptive blind separation of independent signals," Digital Signal Processing, vol. 20, no. 2, pp. 541-551, 2010 